\section{Injection site lesion prevalence and potential risk factors in UK beef cattle}

Elizabeth Cresswell BSc BVMBVS PGCert Farm Animal Practice, MRCVS ${ }^{1}$

John Remnant BVSc CertAVP DipECBHM MRCVS FHEA ${ }^{2}$

Andy Butterworth BSc BVSc PhD CWEL FiBiol FLS DipECAWBM(AWSEL) MRCVS ${ }^{3}$

Wendela Wapenaar DVM PhD DipABVP-Dairy MRCVS, SFHEA ${ }^{2}$

${ }^{1}$ Kyabram Veterinary Clinic, 77 McCormick Road, Kyabram, Victoria, 3620, Australia

${ }^{2}$ School of Veterinary Medicine and Science, University of Nottingham, College Road, Sutton Bonington, Loughborough, Leicestershire, LE12 5RD, UK

${ }^{3}$ School of Veterinary Science, University of Bristol, Langford, BS40 5DU, UK

Corresponding author: Icresswell@kyabramvets.com.au

\section{Abstract}

Injectable veterinary medicinal products (VMPs) are widely used in cattle in the UK, and in particular vaccines are often used on large numbers of animals in the herd. The formation of injection site lesions (ISLs) is a risk when using injectable products, and has potential consequences for meat quality, animal welfare and beef industry income. This study used carcase observation in four abattoirs in England to determine ISL prevalence in beef cattle. Additionally a questionnaire survey was used to investigate vaccination technique amongst UK beef farmers. The ISL prevalence was 4.1\% (95\% confidence interval 3.4-4.9\%). A potential difference between sites being used for vaccination and the distribution of ISLs on carcasses suggested that factors other than vaccination were contributing to ISL incidence. Questionnaire responses highlighted deficits in good vaccination practices such as using the recommended site of injection and needle hygiene. The role of the veterinarian in knowledge transfer is crucial in providing practical injection advice when prescribing vaccines and other VMPs. This study identified factors to address when aiming to reduce ISL formation in UK beef animals.

\section{Keywords}

Injection site lesion, vaccination, compliance, beef, prevalence, carcase 
34 A wide range of injectable veterinary medicinal products (VMPs) are available for use in beef cattle in the UK, including vaccines, antibiotics, anti-inflammatory drugs, mineral supplements and reproductive hormones. Large proportions of these are intended for intramuscular injection and are often administered by farmers. Injections can cause trauma to tissues resulting in an inflammatory response and potential injection site lesions (ISLs) such as cysts, discolouration, nodules or abscesses and subsequent scar tissue (Dexter and others 1994, Van Donkersgoed and others 1999, Roeber and others 2001).

Antibiotics, reproductive hormones and other VMPs are predominantly used on an individual animal basis in beef cattle in the UK, whereas vaccines are usually administered to the whole herd or a managed group within the herd, for example, newly introduced animals or young stock. Seventynine percent of UK beef farmers use vaccines (Cresswell and others, 2014) and it can therefore be assumed that the majority of beef animals passing through abattoirs have been vaccinated during their lives. With the current emphasis on preventative medicine and reduced antibiotic usage in animal production, the use of vaccination is of increasing significance, and it may be considered important to be aware of the associated risks.

Although damage to meat is possible through mechanisms other than injection (e.g. injury), ISLs are of concern due to the loss of quality, value and palatability of meat (George and others 1997). The widespread use of injectable VMPs indicates that ISLs could be a significant reason for carcase trimming. It is hypothesised that the VMP administered, and the subsequent antigenic response, could impact the taste and texture characteristics of the surrounding meat, even if the lesion is trimmed (Van Donkersgoed and others 1997). Injection site lesions in meat could also have implications for human health through toxic residues at injection sites (Reeves, 2007). In addition, compromises to animal welfare through trauma, inflammation and infection should be avoided where possible. Economic loss to the farmer, abattoir and wider industry can result from trimming of lesions from the carcase. This results in lower deadweight payments to the farmer, and also means that meat from valuable cuts, such as the rump area, may have to be discarded. Data from the Food Standards Agency in the UK (FSA) indicated the incidence of abscesses in beef carcasses to be approximately 6\% in 2014 (FSA, 2014); this figure is including intramuscular and hepatic abscesses. In the USA there was a reduction in injection site lesion incidence in beef top sirloin butts from $11 \%$ in 1995 to $2 \%$ in 2000 following national efforts to educate farmers on the losses associated with ISLs. This was achieved through the National Cattlemen's Beef Association instigating Beef Quality Assurance training programs, and publication of guidelines (Appendix 1), with subsequent audits and assessment against these guidelines (Roeber and others 2001). Roeber (2001) estimated that a 9 percentage point reduction in ISLs across a five-year period generated savings of approximately US\$76 $\mathrm{M}$ to the USA meat industry. Although no similar data are available for the UK this suggests that ISLs represent a considerable potential economic loss for the industry.

It is often recommended to inject cattle in the musculature of the neck rather than the rump muscle, as the neck contains meat of lower value (EBLEX, 2013). It has been hypothesised that fewer ISLS occur if injected in the neck due to injected products being restricted to more localised areas by the nature of the anatomy of the fascial planes, and due to the extensive lymphatic supply resulting in rapid absorption (Glock and others 1995). 
In terms of ISLs, intramuscular (IM) injection is of the most concern as the muscle tissue is used for human consumption. However, subcutaneous (SC) vaccines can also lead to muscle damage as described by Van Donkersgoed and others (1999) with respect to clostridial vaccines. Guidelines for administration of VMPs in cattle are provided on datasheets that accompany all licenced VMPs. Previous research showed that compliance with datasheet instructions could be improved; only $33 \%$ of UK cattle farmers who participated in the study referred to the datasheet before administering vaccines (Cresswell and others 2014). Many of the injectable product datasheets recommend using aseptic techniques when injecting cattle, although in practice this is rarely carried out, especially when large numbers of animals are injected, such as during herd vaccination (Cresswell and others 2014). The consequences of not following datasheet instructions have not been well documented in farm animals, but human studies indicate that practices such as poor needle hygiene increase the chance of infection and abscessation (Murphy and others 2001).

The aim of this study was to investigate the prevalence of ISLs in UK beef cattle, and to investigate if, and how, injectable vaccines may contribute to ISLs.

\section{Materials and Methods}

\section{Abattoir}

Four abattoirs were visited between April 2009 and April 2010. The abattoirs selected for the study were a convenience sample, based on throughput of beef cattle only (excluding dairy culls) and their geographical location in England (two in Cumbria, one in Gloucestershire, and one in Somerset). Letters were sent to abattoirs prior to the visit to explain the purpose of the study. Once participation was agreed, the researchers met with the FSA staff (official veterinarian and meat inspectors) and slaughterhouse staff to confirm their past experience in identifying lesions.

Two control points were designated at each abattoir. These were set up at the end of the dressing line immediately after slaughter and in the deboning and butchery area, so that the researchers could observe the routine inspection process without disrupting the normal throughput of carcasses. Inspections were carried out on two consecutive days at each abattoir, for the full duration of the working day. All carcasses on these two days were examined for lesions. Carcasses were inspected visually by FSA and slaughterhouse staff at both control points. Lesions or abnormal tissue were trimmed from the carcase. It was assumed that injection was the most likely cause of these lesions in these commonly used injection sites. The tissue was labelled with date and kill number and a gross morphological description was recorded. The anatomical site of each lesion was recorded and subsequently categorised as per Figure 1 . The ISL samples were stored on ice and taken to the laboratory where they were incised to identify the underlying structures. Lesion samples were photographed and the greatest diameter of each lesion was recorded.

\section{Questionnaire}

A questionnaire was developed and distributed in paper format and online to cattle farmers in the UK between September and November 2011 using a convenience sample. The questionnaire contained 23 questions about cattle vaccine uptake, and collected data on how the vaccines were 
stored and administered. Results not presented in this study are published in Cresswell and others (2014), where a detailed description of the methods is provided.

Respondents were asked what vaccines they used, to identify which vaccine they were most familiar with and answer questions about route of administration, needle usage and instructions regarding that particular vaccine. Respondents were asked to identify the site at which they vaccinate by marking an ' $x$ ' on an image of a cow; the site was subsequently categorised as per Figure 1 . For this study, results from the beef respondents only were used.

Where applicable, vaccine use was categorised as 'correct' or 'incorrect' (e.g. route and site of administration) according to the information provided on the vaccine datasheet (National Office of Animal Health, 2010). 'Datasheet' in this study refers to the information sheet provided with the vaccine, which for most manufacturers in the UK is also published by the National Office of Animal Health (NOAH, 2010). This is an abbreviated form of the Summary of Product Characteristics (SPC), which is the legally approved document for all licenced VMPs available through the Veterinary Medicines Directorate (http://www.vmd.defra.gov.uk/productinformationdatabase/).

\section{Data analysis}

Questionnaire and abattoir data were analysed using Microsoft Excel 2010 (Microsoft, Redmond, USA). A histogram was used to assess the distribution of the size of the lesions to subsequently categorise these as 'small' $(0.1-7.9 \mathrm{~cm})$, 'medium' $(8-15.9 \mathrm{~cm})$ or 'large' $(16-23 \mathrm{~cm})$.

Statistical analysis was carried out in $R$ ( $R$ Core Team, 2013). Fisher's exact test was used to test associations between size and site of lesions. Pearson's Chi-squared test with Yates' continuity correction was used to test associations between site of vaccination and site of lesions. A P-value $\leq 0.05$ was considered significant.

The study received ethical approval from the School of Veterinary Medicine and Science Ethics Committee, The University of Nottingham.

\section{Results}

\section{Abattoir}

Injection site lesion prevalence

2853 beef carcasses were examined and 117 lesions were recorded $(4.1 \%, 95 \%$ confidence interval $3.4 \%-4.9 \%)$.

\section{Site and size of lesions}

Information which described the location on the carcase was available for 93 lesions. This information was not recorded for the remaining 24 lesions as some samples were provided from butchery inspection where the precise carcase location was no longer readily apparent. Out of these, $47 \%(n=44 / 93)$ were located in the rump and $42 \%(n=39 / 93)$ were located in the neck. The remaining $11 \%(n=10 / 93)$ were located in other sites such as the ribs and flank. 
149 Data on the size of the lesion were available for 104 cases. The measured maximum diameter of

150 lesions ranged from 1 to $23 \mathrm{~cm}$. Twenty-three percent were described as small (diameter of 1-

$1517.9 \mathrm{~cm}), 67 \%$ as medium $(8-15.9 \mathrm{~cm})$ and $10 \%$ large $(16-23 \mathrm{~cm})$.

152 There appeared to be larger lesions in the rump compared with the neck, however this was not 153 statistically significant ( $p=0.2$ ) (Table 1 ). Due to information being unavailable from some of the 154 observed lesions, it was only possible to compare lesion size with lesion site for 81 lesions.

Description of lesions

156 A variety of lesion types were identified. Gross morphological descriptions were identified for 76 of 157 the lesions. Many descriptors identified the lesions as being chronic in nature or being the residual 158 and resolving remnants of previous lesions rather than an active infection, e.g. steatosis, scar tissue 159 and fibrosis. Some lesions were specifically described as abscess or pus, suggesting current infection, 160 and less commonly, lesions were identified as cysts, nodules, haemorrhages or melanistic 161 (pigmented).

\section{Questionnaire}

Ninety-two questionnaire respondents classified themselves as beef farmers. Different numbers of respondents were excluded from data analysis of each question due to incomplete responses, for example not identifying a specific vaccine at the start of the questionnaire.

Route and site of vaccine administration

When asked 'Which route of administration did you use for this vaccine?', $56 \%$ ( $n=40 / 72$ ) of respondents used the subcutaneous route and $40 \%(n=29 / 72)$ of respondents used the intramuscular route. For some respondents it was not possible to assess whether the route of administration used was in accordance with the datasheet recommendations because they had not clearly specified which vaccine they were using, or indicated more than one route of injection; these responses were excluded from further analysis. For vaccines to be administered subcutaneously, the correct route of administration was chosen by $86 \%(n=26 / 30)$ of respondents. For intramuscular vaccines, the correct route was chosen by $79 \%(n=19 / 24)$ of respondents.

When asked to indicate the site of injection, significantly more respondents injected in the neck $(p=0.002) ; 60 \%$ of respondents injected in the neck ( $n=40 / 66)$ and $33 \%$ of respondents injected in the rump ( $n=22 / 66$ ) (Figure 1). Thirty-seven responses were excluded from further analysis as the datasheet for the vaccine they were using did not recommend administration in a specific site on the animal, or the respondent had indicated more than one site. Seventy-two percent $(n=21 / 29)$ of respondents administered vaccines in the correct site. administration (60\% in neck, $33 \%$ in rump) and site of lesions observed at the abattoir ( $42 \%$ in neck, $47 \%$ in rump)..

There were 60 respondents who provided answers to both questions regarding route and site of administration. The majority of these respondents vaccinated cattle subcutaneously in the neck $(45 \%, n=27)$, or intramuscularly in the rump $(28 \%, n=17)$. Nine respondents were vaccinating 
intramuscularly in the neck, and two were vaccinating subcutaneously in the rump. The five remaining respondents vaccinated elsewhere on the animal, or indicated to use a non-injectable vaccine.

\section{Needle usage when vaccinating beef cattle}

When asked 'When administering this vaccine by injection, which of the following apply on your farm?' $43 \%$ of respondents started each vaccination session with a new needle or changed needles when they became broken or blunt (Table 2).

\section{Instructions used by farmers when vaccinating beef cattle}

When asked 'What instructions did you follow when administering this vaccine?' $20 \%$ of respondents indicated they did what they had done previously and did not need instructions (Table 3).

\section{Discussion}

The prevalence of ISLS (4.1\%) detected in the abattoir was lower than the FSA national average of approximately $6 \%$ in 2014 . This is to be expected as the FSA data included hepatic abscesses which would not be directly caused by intramuscular or subcutaneous injection. This is higher than the 2.1\% ISL incidence reported in the USA in 2000 (Roeber and others 2001) and represents a significant potential financial loss to the beef industry and a welfare concern through discomfort and pain in the affected animals.

In 2014, 2,669,000 cattle were slaughtered in the UK (AHDB, 2015). If the $4.1 \%$ incidence of ISLS were an unbiased, random representation of animals for the UK it could be extrapolated that between 90,700 and 130,000 beef cattle annually could have ISLs. Although the study sample was a small convenience sample based in four abattoirs, there are no confounding factors to suggest that the prevalence, site and size of ISLs differ from other abattoirs in the UK. Moreover, the ISL prevalence in this study is likely an underestimate of the true prevalence, as lesions could have been missed during inspection as mainly superficial ISLs are observed; Roeber and others (2001) report that ISLs are seldom detected at packing plants because damage is concealed within the muscles and subcutaneous fat.

The proportion of ISLs in the carcasses were similar between the neck (47\%) and rump (42\%) area, whereas the sites at which respondents were vaccinating differed with $60 \%$ vaccinating in the neck and $33 \%$ vaccinating in the rump $(p=0.002)$. Although there was no statistically significant difference in neck-rump proportions between lesions and vaccination sites $(p=0.22)$, investigating a larger sample size may demonstrate significance. Therefore the relevance of factors other than vaccination may be responsible for the formation of ISLs. The two parts of this study were carried out during different time periods (abattoir data from 2009-2010 and questionnaire data during 2011), in two different target populations. However, it is not expected that producer's injection practices and ISL prevalence would have varied significantly during this time.

Large injected volumes of VMPs are thought to produce a greater number of, and larger abscesses and granulation tissue than smaller injected volumes (Van Donkersgoed and others, 1999). It could therefore be hypothesised that antibiotics and anti-inflammatories, which tend to be injected in 
higher volumes in cattle, would be more likely to cause ISLs than vaccines which are injected in relatively small $(2-5 \mathrm{ml})$ volumes. Reproductive hormones are also administered in 2-5 $\mathrm{ml}$ doses but have been demonstrated to be damaging to meat quality in a study using creatine kinase levels as a marker of muscle damage following injection (Fajt and others 2014).

The type and extent of reaction appears to vary between different injectable VMPs and product type may be more significant than product volume - for example, clostridial vaccines are noted for their irritability, readily producing localised damage (Van Donkersgoed and others 1999) with up to $17 \%$ forming abscesses and reactions that persist for 10 week or more (NOAH, 2010), whereas the antimicrobial tilmicosin, administered subcutaneously and in large volumes, did not appear to cause ISLs (Van Donkersgoed and others 2000). It has been proposed that modified live vaccines produce fewer ISLs than killed vaccines, due to the oil-based adjuvants used in killed vaccines (Van Donkersgoed and others 1999, McFarlane and others 1996), although other studies found no difference between ISL incidence using killed or modified live vaccines (Van Donkersgoed and others 2000).

240 VMPs are frequently administered in the rump as this provides a large area that is convenient to inject, often more so than the neck which can be restricted by handling facilities. The economic impact of ISLs may vary depending on which area of the rump is used for injection, i.e. dorsal gluteal muscle vs caudal fold; this study provides limited detail with regards to the precise site indicated by the respondents. Considering the finding of the rump to be such a common injection site, future research should investigate techniques in more detail to accurately assess the impact of losses when using the rump as injection site.

247 Dexter and others (1994) found that $80-90 \%$ of all ISLs were older lesions which is supported by the findings of this study; many of the descriptions suggested that an ISL had occurred prior to slaughter but the active reaction had since resolved. George and others (1995) demonstrated that the time elapsed since injection is not necessarily proportional to the presence of ISLS, and that ISLs were present up to 12 months after (clostridial) vaccination had been carried out. This demonstrates the risk of ISLs when injecting cattle at any time point before slaughter.

This study did not find a relationship between vaccination use and ISLs in beef cattle; however, the findings were limited by the number of questionnaire respondents and the fact that the abattoir data did not originate from cattle produced by questionnaire respondents. However, this initial data highlights the need to improve our understanding of the causes of ISLs. Prospective longitudinal studies identifying which injectable VMPs were administered when, and subsequent carcase inspection would be required to investigate the long-term implications of injecting specific VMPs. Such a study could assess more accurately the relative impact of the widespread use of vaccines in comparison with other VMPs. Whilst this could provide valuable information for veterinarians and farmers on how to avoid ISLs in beef cattle, accurate and systematic data recording on farm is challenging, which will affect the quality of data available for analysis (Velasova and others 2015). The practical and logistical difficulties involved in conducting such a study have so far been prohibitive to the carrying out of such a project. This retrospective study therefore provides an important indication of the current ISL prevalence and potential risk factors in UK beef cattle and supports the need for further investigation into this area. Although selecting VMPs based on their product characteristics (including tendency to form ISLs) is relevant, areas related to compliance, 
such as poor needle usage and site of administration are also within the control of the veterinarian and farmer. A cost-benefit analysis can be made when administering a vaccine; the risk of ISL formation must be balanced with the potential to control disease, which may include financial, welfare and public health benefits, including reduced antimicrobial usage. Vaccination therefore remains important for the UK beef industry and should not be disregarded because of the risk of ISL formation. Instead, efforts should be focused on reducing risk factors, such as addressing why only $72 \%$ of UK beef farmers are using the correct route of administration, or fewer than half of respondents are only changing the needle when broken or blunt.

Using broken or blunt needles increases the chance of causing tissue trauma, resulting in a greater inflammatory reaction (Xie and others 2014). Not changing needles or not employing aseptic techniques increases the chance of transferring pathogens between animals and ISL formation (Niskanen and Lindberg, 2003), which is particularly relevant where vaccination is being used for a whole herd. Fewer than $50 \%$ of respondents were following datasheet recommendations with regard to needle usage and only $40 \%$ consulted the datasheet during a vaccination session. This corroborates a study on BVD vaccination by Meadows (2010) where one third of UK farmers never referred to the datasheet and where overall compliance with datasheet recommendations was poor. Information on vaccine bottles that only provide brief information, were consulted by $56 \%$ of respondents in our study. Comprehensive recommendations for vaccination administration are only available on the datasheet. Vaccination recommendations change and it would therefore be prudent to check the datasheet before a vaccination session. As $95 \%$ of farmers would prefer their information on vaccination to come from their veterinarian (Cresswell and others 2014), he/she has an important knowledge transfer role when prescribing and advising on the use of vaccines in order to increase awareness and minimise the risks of ISL formation. The effect size of the different aspects of non-compliance however are unknown; i.e. whether ISLs are more prone to forming when injecting using a different route or site from the SPC, or whether inappropriate storage or needle hygiene have a greater impact. More research identifying the relative importance of each of these factors will help prioritize areas of focus when engaging with vets and farmers (Cresswell et al., 2013). In addition, for this area of knowledge exchange, there is an opportunity for abattoir findings to be fed back to producers; currently the cost of ISLs is often carried by the abattoir and only in certain cases the farmer is contacted about observed ISLs. To create a feedback loop to the producer will highlight the importance of correct injection techniques to them.

A reduction in ISLS was achieved by the beef industry in the USA through the publication of the National Cattlemen's Beef Association's guidelines. In Australia, the introduction of the Meat Standards Australia grading model to predict consumer scores on meat quality has provided an incentive for producers to improve beef quality (Polkinghorne and others 2008). In the UK the dairy levy board has recently produced guidelines for farmers regarding correct vaccination techniques (AHDB, 2014). However, farmers require individualised approaches when communicating disease prevention programmes (Jansen and others, 2010) and value vaccination advice from their own veterinarian (Cresswell and others 2014). Individual, tailored advice as well as the dissemination of advice from national initiatives is therefore required when aiming to optimise the use of injectable VMPs. This could be provided through theoretical and practical education (e.g. practice newsletters, on-farm advice) and in collaboration with abattoir staff who can provide feedback for the veterinarian and producer on carcase quality and ISL type and location. 
311 As alternatives, non-injectable vaccines, such as oral and intranasal vaccines, can be considered

312 where available. However, non-injectable vaccines are more labour-intensive to apply in cattle,

313 which may be prohibitive in an industry where 'time' is quoted as one of the main barriers to

314 implementing herd health measures (Hall and Wapenaar 2012).

315 This study was partly based on data collected via questionnaires which may have introduced a

316 response bias by attracting responses only from those who have a particular interest in the subject

317 matter (Dohoo and others 2003). In addition, recall bias may have occurred where inaccurate

318 responses could have been provided which do not reflect true vaccination practices occurring on

319 farm. If respondents have an interest in the topic they are likely to be more aware of the risks and

320 are therefore expected to vaccinate correctly. This response bias may have resulted in an

321 overestimation of the reported compliance on UK beef farms. Recall bias may have occurred, and is

322 also more likely to underrepresent occurrence of poor vaccination compliance, as respondents

323 would tend to give the expected answer instead of their true vaccination technique. The

324 convenience sample used for the abattoir data and the practical difficulties encountered in collecting complete lesion data in the abattoirs may have influenced the results; however, in the absence of other published information this study provides an indication of ISL prevalence in beef carcases in the UK.

Conclusion

This study reports a $4.1 \%$ prevalence of ISLs in 2853 carcasses. ISLs have the potential to cause economic loss due to trimming and reduced carcase value and compromise animal welfare and meat quality. Deficits in compliance with recommended injection protocols may be contributing to the occurrence of ISLs. The role of vaccination in the occurrence of ISLs appears limited and is unlikely to be the predominant cause of ISLs. These findings pave the way for prospective longitudinal studies to further investigate the types and causes of ISLs in cattle. Feedback from the abattoir in conjunction with education on compliance with current datasheet recommendations on site of administration and appropriate needle usage from the prescribing veterinarian could have a key role in reducing ISL prevalence.

\section{Acknowledgments}

The authors wish to thank the producers who completed the questionnaire and staff at the abattoirs who participated in the study. In particular, thanks go to Steve Wotton and Andy Grist for their help with lesion sample collection and Amelia Garcia Ara for reviewing the manuscript.

\section{References}

AHDB (AGRICULTURE AND HORTICULTURE DEVELOPMENT BOARD) BEEF AND LAMB, UK YEARBOOK 2015 CATTLE (2015) http://beefandlamb.ahdb.org.uk/wp/wp-content/uploads/2015/07/UKYearbook-2015-Cattle-200715.pdf. Accessed September, 2015

AHDB (AGRICULTURE AND HORTICULTURE DEVELOPMENT BOARD) DAIRY, VACCINATION (2014). http://dairy.ahdb.org.uk/technical-information/animal-health-welfare/vaccination/\#.Vg54E_IViko. 
CRESSWELL, L., RICHENS, I., ARCHER, S., BREEN, J., HUXLEY, J., RANDALL, L., REMNANT, J., WAPENAAR, W., BIGGS, A., KERBY, M. \& STATHAM, J. (2013) Veterinary vaccination advice and perceived farmer compliance on UK dairy farms. Livestock 18, 166-174

CRESSWELL, E., BRENNAN, M. L., BARKEMA, H. W. AND WAPENAAR, W., (2014) A questionnairebased survey on the uptake and use of cattle vaccines in the UK. Veterinary Record Open 1, e000042

DEXTER D.R., COWMAN G.L., MORGAN J.B., CLAYTON R.P., TATUM J.D., SOFOS J.N., SCHMIDT G.R., GLOCK R.D., SMITH G.C. (1994) Incidence of injection-site blemishes in beef top sirloin butts. Journal of Animal Science 72, 4, 824-827

DOHOO I. R, MARTIN S. W., STRYHN H. (2003) Veterinary Epidemiologic Research. 2nd edition. AVC Incorporated. pp208-212

EBLEX (ENGLISH BEEF AND LAMB EXECUTIVE LTD.) (2013) Beef and Sheep BRP 9: Minimising carcase losses for better returns. Agriculture and Horticulture Development Board. Warwickshire, UK.

FAJT, V. R., WAGNER, S. A., PEDERSON, L. L., NORBY, B. (2011) The effect of intramuscular injection of dinoprost or gonadotropin-releasing hormone in dairy cows on beef quality. Journal of Animal Science 89, 6, 1939-1943

FSA (FOOD STANDARDS AGENCY) (2014), Post-mortem reports 2014 (report data was provided by the FSA and is available on request).

GEORGE M.H., TATUM J.D., SMITH G.C., COWMAN G.L. (1997) Injection-site lesions in beef subprimals: incidence, palatability consequences, and economic impact. Compendium on Continuing Education for the Practicing Veterinarian 19, S84-S93

GEORGE M. H., HEINRICH P. E., DEXTER D. R., MORGAN J. B., ODDE K. G., GLOCK R. D., TATUM J. D., COWMAN G. L., SMITH G. C. (1995) Injection-site lesions in carcasses of cattle receiving injections at branding and at weaning. Journal of Animal Science 73, 11, 3235-3240

GLOCK R. D., STANTON T. L., CHENEY J. C., MAXWELL K. W. (1995) Evaluation of tissues response to intramuscular injection of long-acting oxytetracycline. Compendium on Continuing Education for the Practicing Veterinarian 17, S31-S36

GREEN D. S., GREEN M. J., HILLYER M. H., MORGAN K. L. (1987) Injection site reactions and antibody responses in sheep and goats after the use of multivalent clostridial vaccines. Veterinary Record 120(18), 435-439

HALL J., WAPENAAR W. (2012) Opinions and practices of veterinarians and dairy farmers towards herd health management in the UK. Veterinary Record 170, 441

JANSEN J., STEUTEN C. D. M., RENES R. J., AARTS N., LAM T. J. G. M. (2010) Debunking the myth of the hard-to-reach farmer: effective communication on udder health. Journal of Dairy Science 93(3), 1296-1306

MCFARLANE B. J., STOKKA G.L., BASARABA R. (1996) Injection-site reactions to the use of clostridial vaccines. Compendium on Continuing Education for the Practicing Veterinarian 18, S57-S59 
MEADOWS D. (2010) A Study to Investigate the Use and Application of BVDV Vaccine in UK Cattle. Cattle Practice 18(3), 202-15

MURPHY E. L., DEVITA D., LIU H., VITTINGHOFF E., LEUNG P., CICCARONE D. H., EDLIN B. R. (2001) Risk factors for skin and soft-tissue abscesses among injection drug users: a case-control study. Clinical Infectious Diseases 33(1), 35-40

NATIONAL OFFICE OF ANIMAL HEALTH (2010) Compendium of Data Sheets for Animal Medicines. National Office of Animal Health Ltd. pp1-1100

NISKANEN, R AND LINDBERG, A. (2003) Transmission of bovine viral diarrhoea virus by unhygienic vaccination procedures, ambient air and from contaminated pens. The Veterinary Journal 165, 125130

POLKINGHORNE R., THOMPSON J. M., WATSON R., GEE A., PORTER M. (2008) Evolution of the Meat Standards Australia (MSA) beef grading system. Australian Journal of Experimental Agriculture 48 1351-1359

R CORE TEAM (2013) R: A language and environment for statistical computing. Vienna, Austria: R Foundation for Statistical Computing. http://www.R-project.org.

REEVES, P.T. (2007) Residues of veterinary drugs at injection sites. Journal of Veterinary Pharmacology and Therapeutics 30, 1-17

ROEBER D.L., CANNELL R.C., WAILES W.R., BELK K.E., SCANGA J.A., SOFOS J.N., COWMAN G.L., SMITH G.C. (2002) Frequencies of Injection-Site Lesions in Muscles From Rounds of Dairy and Beef Cow Carcasses. Journal of Dairy Science 85, 532-536

ROEBER D. L., CANNELL R. C., BELK K. E., SCANGA J. A., COWMAN G. L., SMITH G. C. (2001) Incidence of injection-site lesions in beef top sirloin butts. Journal of Animal Science 79(10), 2615-8

VAN DONKERSGOED J., DIXON S., BRAND G., VANDERKOP M. (1997) A survey of injection site lesions in fed cattle in Canada. Canadian Veterinary Journal 38(12), 767-72

VAN DONKERSGOED J., VANDERKOP M., SALISBURY C., SEARS L., HOLOWATH J. (1999) The effect of administering long-acting oxytetracycline and tilmicosin either by dart gun or by hand on injection site lesions and drug residues in beef. Canadian Veterinary Journal 40, 583-587

VAN DONKERSGOED J., DUBESKI P. L., VANDERKOP M., AALHUS J. L., BYGROVE S., STARR W. N. (2000) The effect of animal health products on the formation of injection site lesions in subprimals of experimentally injected beef calves. The Canadian Veterinary Journal 41(8), 617-622

VELASOVA M., DREWE J. A., GIBBONS J., GREEN M., GUITIAN J. (2015) Evaluation of the usefulness at national level of the dairy cattle health and production recording systems in Great Britain. Veterinary Record 177, 304

XIE Y. M., SHANQING X., ZHANG C. S., XUE C. C. (2014) Examination of surface conditions and other physical properties of commonly used stainless steel acupuncture needles. Acupuncture in Medicine $32,146-154$ 
452

453

454

455

456

457

458

459

460

461

462

463

464

465

466

467

468

469

470

471

472

473 
Table 1: Size of injection site lesions found in the rump and neck $(n=72)$. The site of lesions found elsewhere on the carcasses $(n=9)$ are not specified in this table.

\begin{tabular}{|l|l|l|lr|}
\hline Size & $\begin{array}{l}\text { Total number of lesions } \\
\text { found on carcasses }(n=81)\end{array}$ & Rump $(n=37)$ & Neck $(n=35)$ \\
\hline Small $-0.1-7.9 \mathrm{~cm}$ & 22 & 10 & 10 & 476 \\
\hline Medium $-8-15.9 \mathrm{~cm}$ & 53 & 22 & 24 & 478 \\
\hline Large $-16-23 \mathrm{~cm}$ & 6 & 5 & 1 & 479 \\
\hline
\end{tabular}

480

481

482

483

484

485

486

487

488

489

490

491

492

493

494

495

496

497

498

499

500

501

502 
Table 2: Frequency of each of the answers to the question 'When administering this vaccine by injection (using e.g. a syringe, vaccinator gun), which of the following apply on your farm? (Please tick all that apply)' ( $n=69)$.

\begin{tabular}{|l|l|}
\hline Answer & Responses ( $\mathbf{n})$ \\
\hline A new needle is used to start the vaccination session & $43 \%(30)$ \\
\hline Needle is changed when broken/blunt & $43 \%(30)$ \\
\hline $\begin{array}{l}\text { A different needle is used for injecting animals and } \\
\text { drawing up vaccine from the bottle }\end{array}$ & $41 \%(28)$ \\
\hline $\begin{array}{l}\text { A new needle is used between each different group of } \\
\text { animals }\end{array}$ & $27 \%(19)$ \\
\hline A new needle is used between each vaccine bottle & $26 \%(18)$ \\
\hline Other (please specify)* & $9 \%(6)$ \\
\hline A new needle is used for each animal & $6 \%(4)$ \\
\hline
\end{tabular}

* 'After 5 or 6 animals', 'approx every 10 cattle', 'a new needle is used after every 5 animals', 'new injecting needle every 3 or 4 animals', ' 1 needle for large groups from same source with same vaccine, but needle disposed of after each batch' and 'I only have around 10 animals, so one group and needle doesn't get blunt'. 
Table 3: Frequency of each of the answers to the question 'What instructions did you follow when 543 administering this vaccine? (Please tick all that apply)' ( $n=72)$.

\begin{tabular}{|l|l|}
\hline Answer & Responses ( $\mathbf{n}$ ) \\
\hline I followed the instructions on the vaccine box/bottle & $56 \%(40)$ \\
\hline I followed the instructions on the enclosed datasheet & $40 \%(29)$ \\
\hline $\begin{array}{l}\text { I did what I have done previously and did not need } \\
\text { instructions }\end{array}$ & $20 \%(21)$ \\
\hline $\begin{array}{l}\text { I followed the verbal instructions given by my vaccine } \\
\text { supplier }\end{array}$ & $14 \%(10)$ \\
\hline I followed the instructions on the dispensing label & $6 \%(4)$ \\
\hline $\begin{array}{l}\text { I followed the written instructions given by my } \\
\text { vaccine supplier }\end{array}$ & $6 \%(4)$ \\
\hline $\begin{array}{ll}\text { Other (please specify)* } \\
\text { * 'Administered by vet' }\end{array}$ & $1 \%(1)$ \\
\hline
\end{tabular}


Figure 1. Diagram on which respondents were asked to indicate with an ' $x$ ' where they would

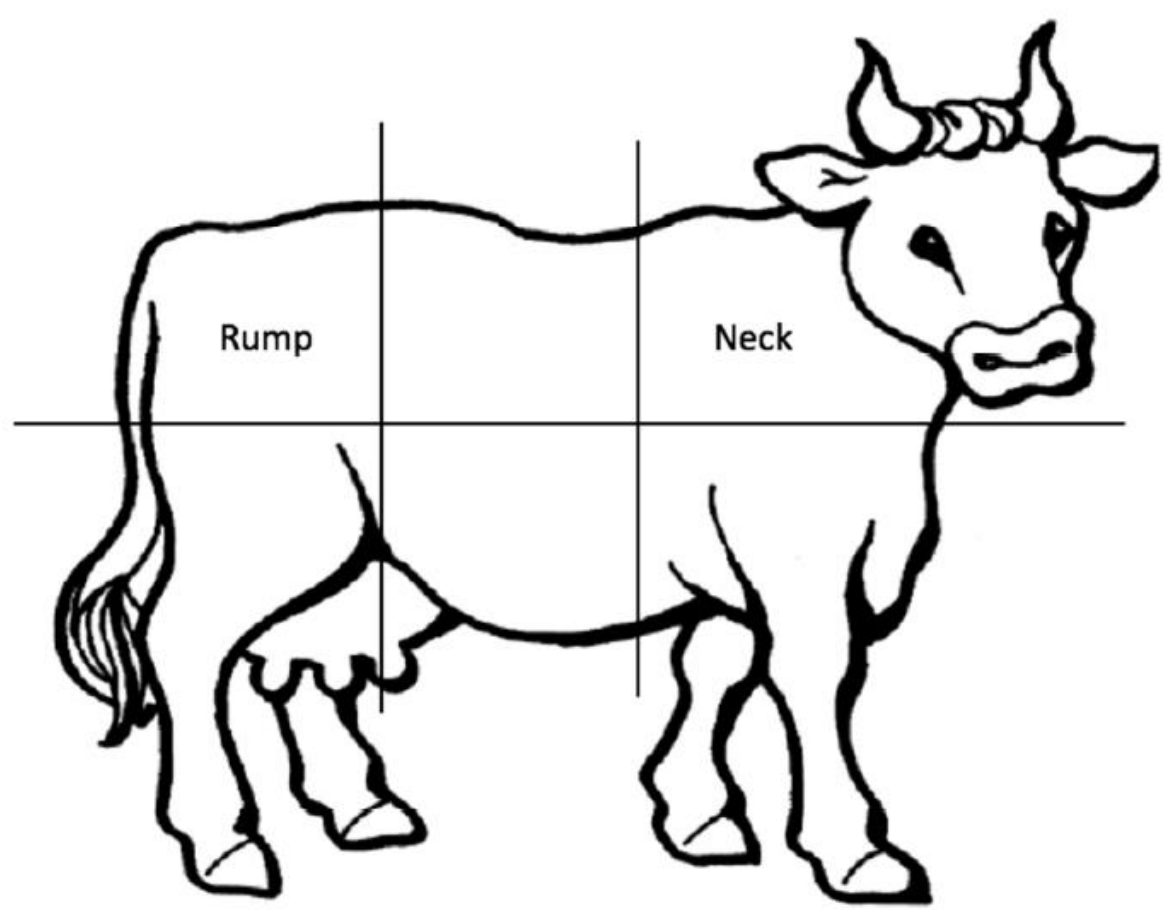

Caption : Fiqure 1. Diagram on which respondents were asked to indicate with an ' $x$ ' where they would vaccinate their cattle. Subsequently the diagram was split into six sections. 